\title{
Human ESC-Derived MSCs Outperform Bone Marrow MSCs in the Treatment of an EAE Model of Multiple Sclerosis
}

\author{
Xiaofang Wang \\ University of Connecticut School of Medicine and Dentistry \\ Kumiko Ijichi \\ University of Connecticut School of Medicine and Dentistry \\ Debayon Paul \\ University of Connecticut School of Medicine and Dentistry \\ Adam S. Lazorchak \\ University of Connecticut School of Medicine and Dentistry \\ Joel S. Pachter \\ University of Connecticut School of Medicine and Dentistry \\ See next page for additional authors
}

Follow this and additional works at: https://opencommons.uconn.edu/uchcres_articles

Part of the Life Sciences Commons, and the Medicine and Health Sciences Commons

\section{Recommended Citation}

Wang, Xiaofang; Ijichi, Kumiko; Paul, Debayon; Lazorchak, Adam S.; Pachter, Joel S.; Crocker, Stephen J.; and Xu, Ren-He, "Human ESC-Derived MSCs Outperform Bone Marrow MSCs in the Treatment of an EAE Model of Multiple Sclerosis" (2014). UCHC Articles - Research. 244.

https://opencommons.uconn.edu/uchcres_articles/244 


\section{Authors}

Xiaofang Wang, Kumiko Ijichi, Debayon Paul, Adam S. Lazorchak, Joel S. Pachter, Stephen J. Crocker, and Ren-He Xu 


\title{
Stem Cell Reports
}

Article

\section{Human ESC-Derived MSCs Outperform Bone Marrow MSCs in the Treatment of an EAE Model of Multiple Sclerosis}

\author{
Xiaofang Wang,,$^{1,2,7}$ Erin A. Kimbrel,,$^{3,7}$ Kumiko Ijichi, ${ }^{4}$ Debayon Paul, ${ }^{5}$ Adam S. Lazorchak, ${ }^{2}$ Jianlin Chu, ${ }^{3}$ \\ Nicholas A. Kouris, ${ }^{3}$ Gregory J. Yavanian, ${ }^{3}$ Shi-Jiang Lu, ${ }^{3}$ Joel S. Pachter, ${ }^{5}$ Stephen J. Crocker, ${ }^{4}$ Robert Lanza, ${ }^{3, *}$ \\ and Ren-He $\mathrm{Xu}^{1,2,6, *}$ \\ ${ }^{1}$ Department of Genetics and Developmental Biology, University of Connecticut Health Center, Farmington, CT 06030, USA \\ 2ImStem Biotechnology, Inc., 400 Farmington Avenue, Farmington, CT 06030, USA \\ ${ }^{3}$ Advanced Cell Technology, 33 Locke Drive, Marlborough, MA 01752, USA \\ ${ }^{4}$ Department of Neuroscience, University of Connecticut Health Center, Farmington, CT 06030, USA \\ ${ }^{5}$ Department of Cell Biology, University of Connecticut Health Center, Farmington, CT 06030, USA \\ ${ }^{6}$ Faculty of Health Sciences, University of Macau, Taipa, Macau, China \\ ${ }^{7}$ Co-first author \\ *Correspondence: renhe.xu@imstem.com (R.-H.X.), rlanza@advancedcell.com (R.L.) \\ http://dx.doi.org/10.1016/j.stemcr.2014.04.020 \\ This is an open access article under the CC BY-NC-ND license (http://creativecommons.org/licenses/by-nc-nd/3.0/).
}

\section{SUMMARY}

Current therapies for multiple sclerosis (MS) are largely palliative, not curative. Mesenchymal stem cells (MSCs) harbor regenerative and immunosuppressive functions, indicating a potential therapy for MS, yet the variability and low potency of MSCs from adult sources hinder their therapeutic potential. MSCs derived from human embryonic stem cells (hES-MSCs) may be better suited for clinical treatment of MS because of their unlimited and stable supply. Here, we show that hES-MSCs significantly reduce clinical symptoms and prevent neuronal demyelination in a mouse experimental autoimmune encephalitis (EAE) model of MS, and that the EAE disease-modifying effect of hES-MSCs is significantly greater than that of human bone-marrow-derived MSCs (BM-MSCs). Our evidence also suggests that increased IL-6 expression by BM-MSCs contributes to the reduced anti-EAE therapeutic activity of these cells. A distinct ability to extravasate and migrate into inflamed CNS tissues may also be associated with the robust therapeutic effects of hES-MSCs on EAE.

\section{INTRODUCTION}

Multiple sclerosis (MS) is a chronic neuroinflammatory disease characterized by infiltration of peripheral immune cells into the CNS through an impaired blood-brain barrier (BBB) or blood-spinal cord barrier (BSCB), and loss of myelin with accompanying scarring of axons (McFarland and Martin, 2007). However, most current treatments for MS only offer palliative relief without providing a cure, and many are also associated with adverse effects that limit their long-term utility (Weber et al., 2012).

Transplantation of mesenchymal stem/stromal cells (MSCs) for the treatment of MS has emerged as an attractive therapy due to the immunomodulatory and neuroregenerative properties of these cells (Auletta et al., 2012; Pittenger et al., 1999) and their potential ability to repair the BBB (Chao et al., 2009) with fewer side effects (Lalu et al., 2012). MSC can home to injured tissues and exert therapeutic effects through the secretion of immunomodulatory and trophic factors as well as through direct cell-cell contact (Uccelli and Prockop, 2010). Importantly, allogeneic MSCs generally do not provoke a strong host immune response due to lack of expression of immune costimulatory receptors and low expression of major histocompatibility complex (MHC) class II antigens (Uccelli and Prockop, 2010), raising the possibility that cells derived from a single donor may be used to treat a large number of patients.

Human adult-tissue-derived MSCs have shown therapeutic utility in experimental autoimmune encephalitis (EAE) models of MS (Bai et al., 2009; Gordon et al., 2008, 2010; Peron et al., 2012; Zhang et al., 2005) and in clinical trials for MS patients (Connick et al., 2012; Karussis et al., 2010; Mohyeddin Bonab et al., 2007; Yamout et al., 2010); however, the large variability in the efficacy of MSCs hinders their development as a standard MS therapy. Extensive in vitro expansion of MSCs may diminish the efficacy of these cells (Kyriakou et al., 2008), and MSCs derived from younger cell sources (e.g., embryonic, fetal, and umbilical cells) have higher in vitro proliferation potential and can more readily differentiate (Barlow et al., 2008; Giuliani et al., 2011). Thus, deriving MSCs from a young and renewable (i.e., pluripotent) cell source, such as human embryonic stem cells (hESCs), could (1) alleviate the quantity and quality issues involved in the use of adulttissue-derived MSCs, (2) obviate the need for constant donor recruitment, and (3) reduce the risk of pathogenicity from the use of multiple donors.

Different groups have derived MSCs from hESCs (hESMSCs) with a morphology and immunophenotype similar to those of BM-MSCs. Previously described derivation methods involve coculturing with mouse OP9 cells and 
A

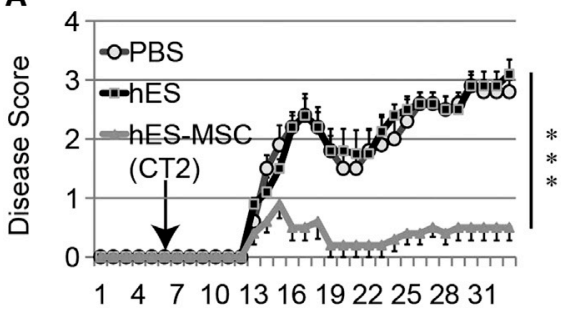

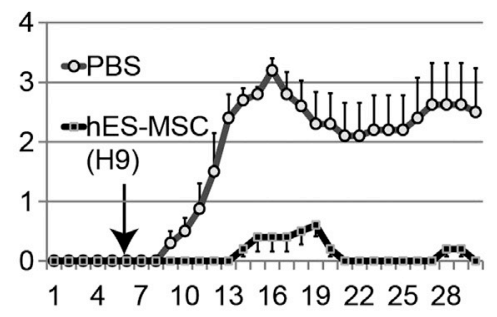

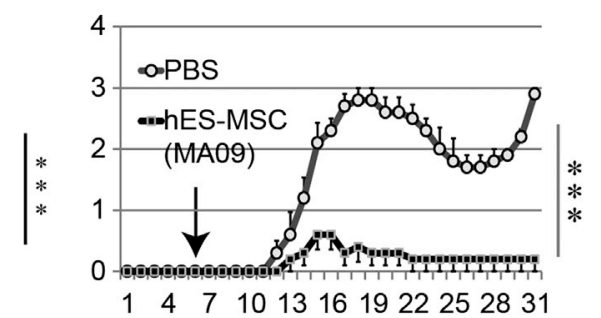

Day Post-immunization

B
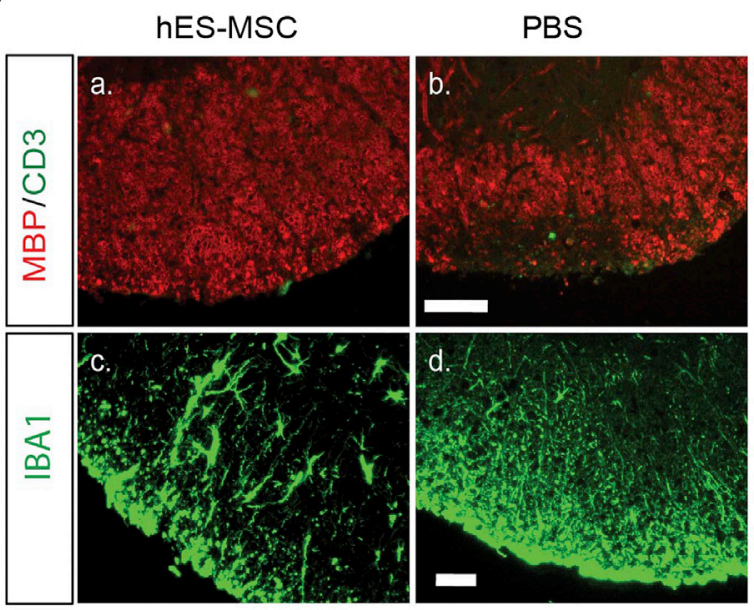

C

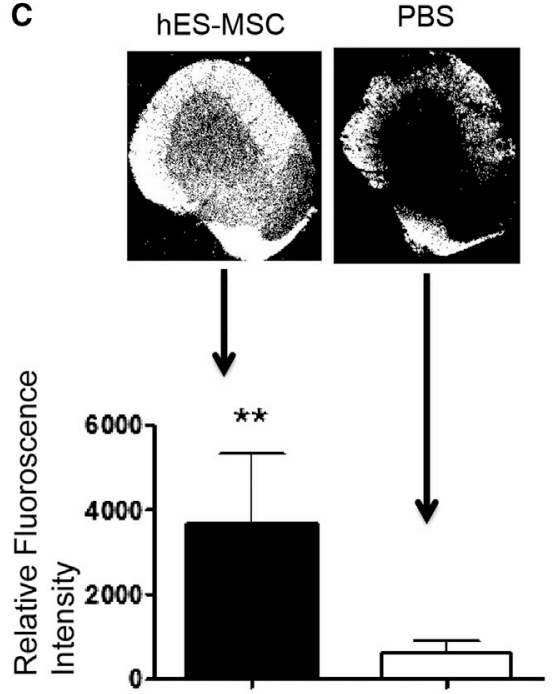

D

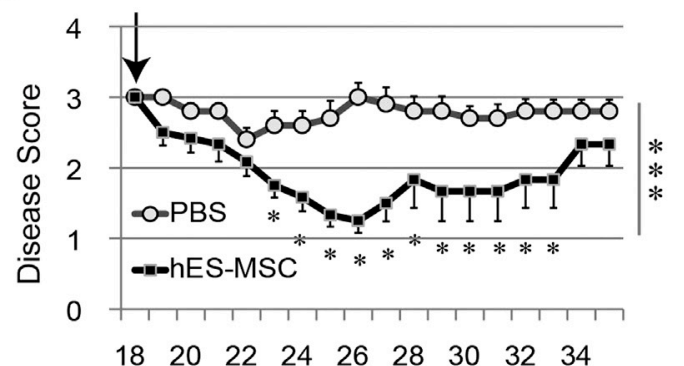

Day Post-immunization

Figure 1. hES-MSCs Attenuate the Disease Score of $\mathrm{MOG}_{35-55}$ /CFA-Immunized Mice

(A) Disease scores of $\mathrm{MOG}_{35-55}$ /CFA-immunized mice treated with $10^{6}$ hES-MSCs (CT2, H9, and MA09) or $10^{6}$ parental hESCs at day 6 postimmunization. $n=5,{ }^{* *} p<0.001$ by Mann-Whitney. Error bar, SEM.

(B) Immunohistochemical detection of MBP (red) and CD3 for T cells (green) (a and b) and IBA1 for microglia (green) (c and d) on lumbar spinal cord cross-sections from MOG $_{35-55}$ /CFA-immunized mice treated with either hES-MSC ( $a$ and $c$ ) or PBS (b and d). Scale bars, $250 \mu \mathrm{m}$ ( $a$ and $b$ ) and $120 \mu \mathrm{m}$ (c and d).

(C) Relative fluorescent intensity measurements of MBP expression in digitally captured spinal cord hemi-sections. $n=4-6,{ }^{* *} p<0.02$. Error bar, SD. The regions shown are from the lumbar level of the spinal cord. Error bars indicate SD and the $p$ value was determined using two-tailed unpaired Student's t test of the total fluorescence signal of each section. 
sorting, scraping, or handpicking of cells (Barberi et al., 2005; Brown et al., 2009; Gruenloh et al., 2011; Hwang et al., 2008; Olivier et al., 2006; Vodyanik et al., 2010), which limits the efficiency and purity of the hES-MSCs, as well as the ability to scale up their production. hESMSCs have been used in some disease models, such as inflammatory bowel disease, lupus, and uveitis (Kimbrel et al., 2014; Sánchez et al., 2011); however, no one has shown whether hES-MSCs can be used to treat an EAE model of MS or compared the immunosuppressive functions of hES-MSCs and BM-MSCs. Here, using an improved hemangioblast-enriching method ( $\mathrm{Lu}$ et al., 2007), we generated hES-MSCs from the MA09 ESC line (Kimbrel et al., 2014) and multiple other hESC lines. We demonstrate that these hES-MSC lines can effectively treat an EAE model of MS and outperform multiple lines of BMMSCs in therapeutic activities.

\section{RESULTS}

hES-MSCs Attenuate EAE Disease in MOG $_{35-55} /$ CFAImmunized Mice when Administered either Prophylactically or Therapeutically

In this study, we derived MSCs through a hemangioblastenriched, intermediate stage as described previously (Kimbrel et al., 2014). We tested the reproducibility of this method by generating independent MSC lines from four different hESC lines: H9 (Thomson et al., 1998), CT2 (derived at UConn; Wang et al., 2009), MA09 (Klimanskaya et al., 2006), and ES03-Envy (Envy, a GFP ${ }^{+}$line derived at ES International; Costa et al., 2005). These hES-MSC lines expressed cell surface markers consistent with those of adult human MSCs (Figure S1A available online) and were capable of differentiating into osteocytes, adipocytes, and chondrocytes (Figure S1B). We also confirmed that the hES-MSCs were karyotypically normal for at least 12 passages (Figure S1C) and did not express telomerase (Figure S1D).

We employed a standard EAE model of MS in which C57BL/6 mice were immunized with an emulsion of MOG $_{35-55}$ peptide and complete Freund's adjuvant (CFA) to test the therapeutic utility of our hES-MSC lines. Six days after immunization but prior to disease onset, mice were injected with $1 \times 10^{6} \mathrm{hES}$-MSCs or PBS intraperitoneally (i.p.). hES-MSCs derived from three hESC lines (CT2, MA09, and H9) all significantly attenuated the daily (Fig- ure 1A), cumulative, and maximal disease scores (Figure S1E). H9 hES-MSCs also delayed the disease onset, and MA09 hES-MSCs appeared to lower the disease incidence (Figure S1E). However, mice injected with parental hESCs (CT2) manifested high disease scores similar to those of PBS controls (Figure 1A). To confirm the results, we histologically analyzed microglial inflammatory activity within the spinal cord. Immunostaining for ionized calcium-binding adaptor molecule 1 (IBA1) revealed inhibited development of microgliosis in $\mathrm{MOG}_{35-55} / \mathrm{CFA}-$ immunized mice treated with hES-MSCs compared with those treated with PBS (Figure 1B). Infiltration of $\mathrm{CD}^{+}$ $\mathrm{T}$ cells into the spinal cord was decreased and the number of interleukin-17 (IL-17)- and interferon $\gamma$ (IFN $\gamma$ )-expressing $\mathrm{CD}^{+} \mathrm{T}$ cells in the CNS was also decreased by hES-MSC treatment (Figures 1B, S1F, and S1G). Stronger immunostaining for myelin-binding protein (MBP) suggests that demyelination was prevented in mice treated with hESMSCs (Figures 1B and 1C).

We also tested the effect of hES-MSC treatment on mice that had already developed EAE (postonset). hES-MSCs were injected on day 18 postimmunization, when all mice had disease scores of 3 . We observed a gradual decline in disease scores from 3 down to an average score of 1.7 by day 30 in hES-MSC-treated mice, whereas the PBS-treated mice showed an average score of 2.8 by day 30 (Figure 1D). Collectively, the data presented in Figure 1 show that hESMSCs can reproducibly decrease disease severity both prophylactically and therapeutically in the mouse EAE model.

\section{Mitotically Arrested hES-MSCs Retain the EAE- \\ Inhibitory Effect}

MSCs transplanted into animals may undergo malignant transformation or support tumor growth formed by host cells (Djouad et al., 2003; Wong, 2011). However, since short-term cytokine secretion and cell-cell contact may be sufficient to exert MSC functions (Uccelli and Prockop, 2010), we hypothesized that mitotically arrested MSCs may still execute a disease-modifying effect. To test this, we irradiated hES-MSCs at 80 Gy immediately before injecting cells into $\mathrm{MOG}_{35-55} / \mathrm{CFA}$-immunized mice at day 6 postimmunization. This irradiation regimen did not significantly reduce the viability of hES-MSCs that were replated and cultured for $48 \mathrm{hr}$ in vitro $(90 \%$ of the cells were trypan-blue negative), but was sufficient to completely attenuate cell proliferation as assessed by

(D) Disease scores of $\mathrm{MOG}_{35-55} /$ CFA-immunized mice treated with $10^{6} \mathrm{hES}-\mathrm{MSC}$ 18 days postimmunization. $\mathrm{n}=6,{ }^{* * *} \mathrm{p}<0.001$ by MannWhitney. Error bar, SEM. At day 17 postimmunization, all mice with a disease score of 3 were pooled into a single group and then randomly assigned to either the PBS-treatment group or the hES-MSC-treatment group. Mice that were immunized but did not show a disease score of 3 on day 17 were removed from the study.

See also Figure S1. 
A

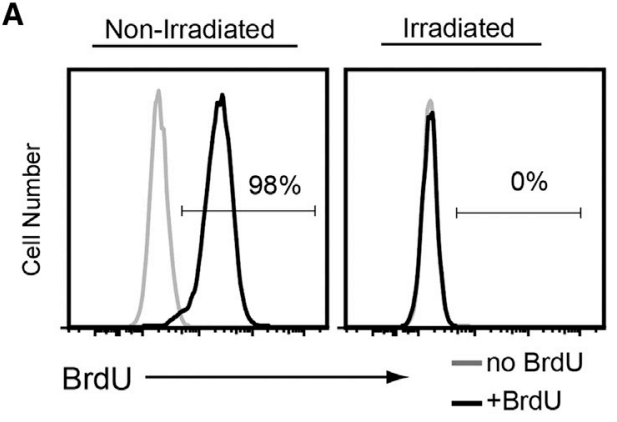

B

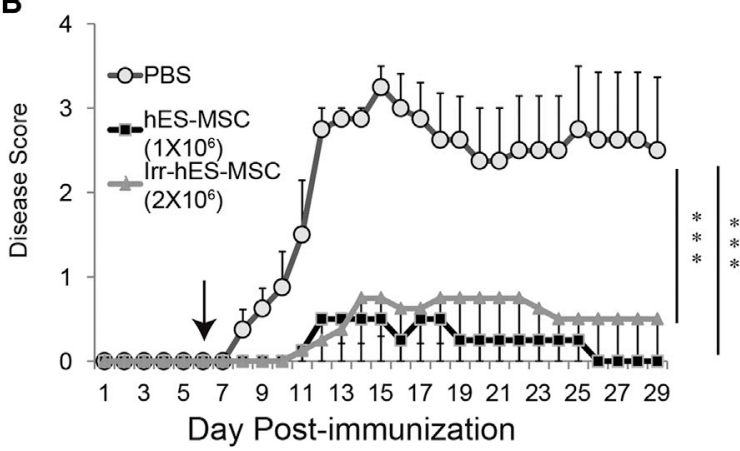

C
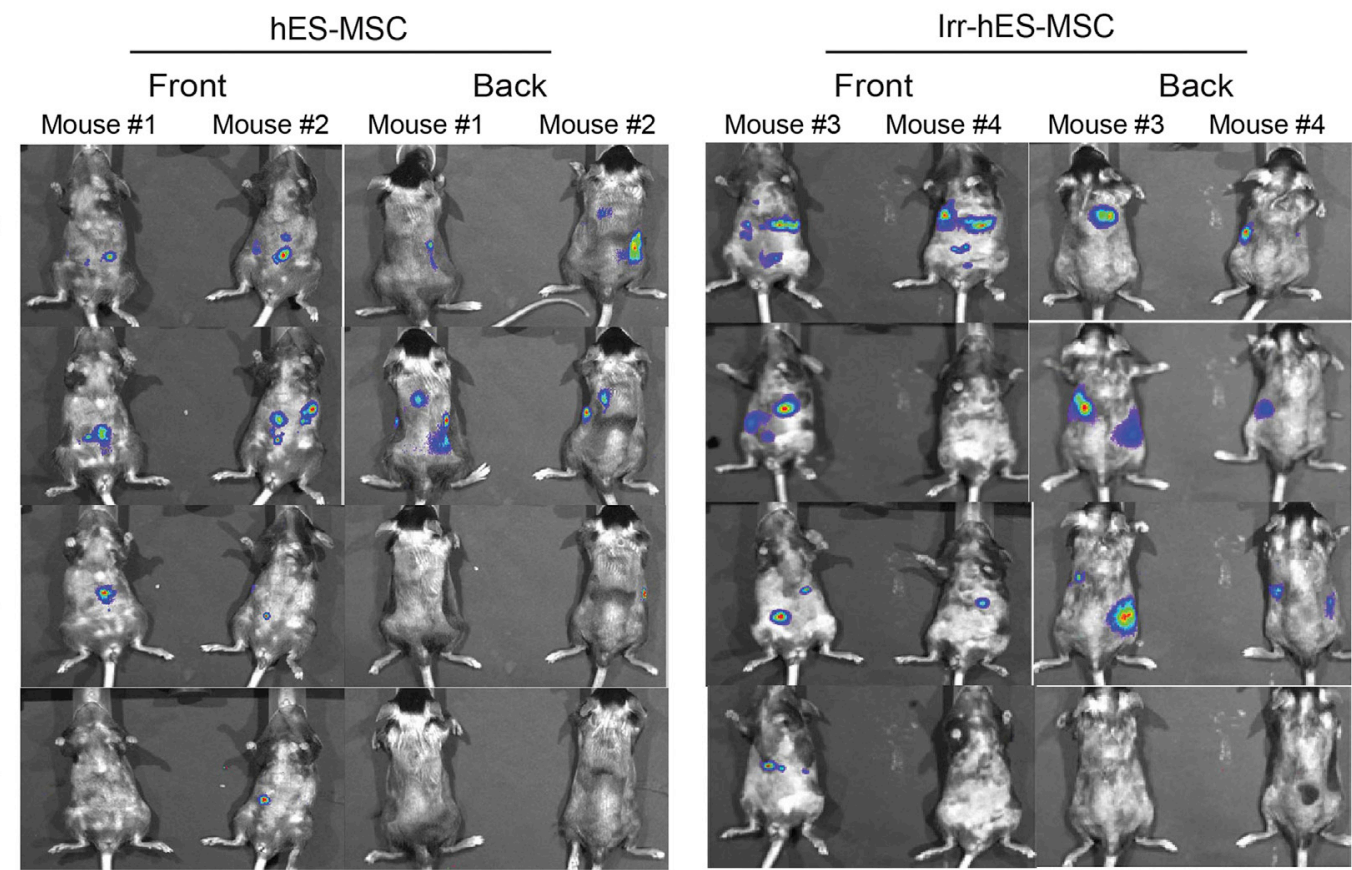

Figure 2. Mitotically Arrested hES-MSCs Retain the EAE-Inhibitory Effect

(A) Nonirradiated or irradiated hES-MSCs $\left(1 \times 10^{5}\right)$ were cultured in vitro for $48 \mathrm{hr}$ in the absence (gray line) or presence (black line) of BrdU. The percentage of proliferating cells, as determined by flow-cytometry staining for BrdU ${ }^{+}$cells, is indicated.

(B) Disease scores of $\mathrm{MOG}_{35-55}$ /CFA-immunized mice treated at day 6 with PBS, $10^{6}$ nonirradiated or $2 \times 10^{6}$ irradiated hES-MSCs (MA09); $\mathrm{n}=4,{ }^{* *} \mathrm{p}<0.001$ by Mann-Whitney for comparison with the PBS control. Error bar, SEM. The table below shows a comparison of cumulative disease score (Cum. D.S.), maximum disease score (Max. D.S.), disease incidence, and disease onset day.

(C) Nonirradiated (left) and irradiated (right) luciferase-expressing hES-MSCS (CT2) were tracked in MOG $_{35-55}$ /CFA-immunized mice by in vivo bioluminescence imaging using the Xenogen IVIS 100 system.

See also Figure S2.

bromodeoxyuridine (BrdU) incorporation during this same time period (Figure 2A). Mice that received $2 \times 10^{6}$, but not $1 \times 10^{6}$, irradiated cells showed a similar reduction in EAE disease severity as mice who received $1 \times 10^{6}$ nonirradiated
hES-MSC (Figures 2B and S2A). To determine the lifespan of irradiated hES-MSC in vivo, we established a CT2 hESC clone with constitutive expression of luciferase in the hESCs and subsequent hES-MSCs (Figure S2B) by 
transducing the cells with a lentiviral vector (Pomper et al., 2009). Using these luciferase-expressing hES-MSCs, we found that both irradiated and nonirradiated hES-MSCs had roughly the same lifespan of at least 7 days in wildtype mice as determined by whole-body bioluminescence imaging (Figure 2C).

Teratoma formation is another concern for any cells differentiated from pluripotent cells. To assess this risk, we injected hES-MSCs into immunodeficient SCID-beige mice at $1 \times 10^{6}$ cells/mouse, and found no tumor formation at the injection sites within 2 months, whereas teratomas formed in mice injected with the same dose of parental hESCs (data not shown).

\section{hES-MSCs Have Stronger EAE-Inhibitory Effects than BM-MSCs}

Next, we compared hES-MSCs and BM-MSCs in the prophylactic EAE model. We derived MSCs from six different BM donors (four from frozen monocytes [MNCs] and two from fresh $\mathrm{BM}$ ) and obtained two BM-MSC lines at passage 1 from the Texas A\&M MSC repository. We tested BM-MSC lines anywhere from passage 2 to 4 and found that none of them could consistently attenuate EAE disease scores of $\mathrm{MOG}_{35-55} / \mathrm{CFA}$-immunized mice, as shown in Figures $3 \mathrm{~A}$ (BM-MSC\#1-3), 3B (BM-MSC\#4), 6 (GFP-expressing BMMSC\#5), and S3F (BM-MSC\#7-8). Of note, one BM-MSC line (BM-MSC\#6, from Texas A\&M) showed moderate but significant disease-modifying effects (Figure $3 \mathrm{C}$ ) at passage 2 relative to control PBS-treated mice $(\mathrm{p}<0.001)$, but these effects were gone at passage 4 (see Figure $5 \mathrm{~F}$ ). This is in marked contrast to the four independent hES-MSC lines that all showed a strong disease-inhibitory effect when tested up to passage 5, as shown in Figures 1A (H9, CT2, and MA09) and 6 (GFP-expressing Envy).

$\mathrm{EAE} / \mathrm{MS}$ is accompanied by infiltration of reactive T cells into the CNS (McFarland and Martin, 2007). We found that hES-MSC-injected mice had significantly fewer $\mathrm{CD} 4^{+}$and $\mathrm{CD}^{+} \mathrm{T}$ cell infiltrates in the CNS, including Th1 and Th17 $\mathrm{CD}^{+}$subsets, than PBS-treated $\mathrm{MOG}_{35-55} / \mathrm{CFA}-$ immunized mice (Figures 3D, S1F, and S1G), whereas parental hESC line CT2-treated mice had levels comparable to those in controls (Figure S1F). In contrast, BM-MSCtreated mice actually displayed significantly more $\mathrm{CD}^{+}$ and slightly more $\mathrm{CD}^{+} \mathrm{T}$ cell infiltrates than PBS-treated MOG $_{35-55} /$ CFA-immunized mice (Figure 3D, first two panels). This included similar or greater Th1 numbers and consistently greater Th17 numbers compared with the controls (Figure 3D, last two panels). Reduced FluoroMyelin staining of MBP in the spinal cord of both PBS- and BM-MSC-treated mice suggests severe demyelination, whereas MBP levels were preserved in the spinal cord of hES-MSC-treated mice (Figure 3E). The damaged regions in BM-MSC-treated mice also show a high number of
DAPI-positive cells (Figure 3E), suggesting more inflammatory cell infiltration. To determine whether the reduced FluoroMyelin staining in the BM-MSC-treated mice was due to reduced levels of myelin, loss of entire axons, or inflammatory infiltrate blocking myelin staining, we performed costaining for MBP and neurofilament (NF) on day 32 lumbar spinal cord cross-sections. Quantification of MBP-positive areas again revealed significantly lower levels of myelination in BM-MSC-treated mice as compared with hES-MSC-treated ones, whereas NF staining revealed similar numbers of axons in both groups (Figures S3A-S3E).

Considering the important role of regulatory $\mathrm{T}$ cells $\left(\mathrm{T}_{\text {reg }}\right.$ cells detected as $\mathrm{CD}^{+}$, $\mathrm{Foxp}^{+}$, and $\mathrm{CD} 25^{+}$) in suppressing inflammation, we examined the ratio of $\mathrm{T}_{\text {reg }}$ cells among infiltrated $\mathrm{CD}^{+}{ }^{+} \mathrm{T}$ cells in the $\mathrm{CNS}$, and found no difference in hES-MSC-treated versus control $\mathrm{MOG}_{35-55} / \mathrm{CFA}$-immunized mice (Figure S3G). This is similar to a previous report (Zappia et al., 2005). In vitro, both hES-MSCs and BMMSCs increased $\mathrm{T}_{\text {reg }}$ cell proliferation in the presence of IL-2, but there was no remarkable difference between the two groups (Figure $\mathrm{S} 3 \mathrm{H}$ ). This suggests that enhanced $\mathrm{T}_{\text {reg }}$ cell proliferation is a common response to both hESMSCs and BM-MSCs, but is not necessarily a contributor to their divergent effects in the in vivo EAE model.

Analyzing MSC Effects on T Cell Functions: hES-MSCs Show Stronger Inhibition of Th1 Differentiation than BM-MSCs

We next compared hES-MSCs and BM-MSCs for their ability to inhibit $\mathrm{T}$ cell proliferation in vitro, using mixed leukocyte reaction (MLR) assays. We incubated carboxyfluorescein succinimidyl ester (CFSE)-labeled mouse naive Tcells isolated from lymph nodes with increasing amounts of BM-MSCs or hES-MSCs. Both types of MSCs inhibited $\mathrm{CD}^{+}$and $\mathrm{CD}^{+}{ }^{+}$cell proliferation in response to a constant amount of anti CD3/anti-CD28 stimulation to similar degrees (Figure 4A). Likewise, BM-MSCs and hES-MSCs similarly inhibited human peripheral blood mononucleated cell (PBMC) proliferation induced in response to phytohemagglutinin (PHA) over a range of PBMC/MSC ratios (Figure 4B). Together, these data suggest that BMMSCs and hES-MSCs display similar inhibitory effects on $\mathrm{T}$ cell proliferation in vitro.

Since $\mathrm{MOG}_{35-55} / \mathrm{CFA}$-immunized mice treated with BMMSCs had more Th1 and Th17 cell infiltration in the CNS than mice treated with hES-MSC (Figure 3D), we examined these $\mathrm{T}$ cell subtypes in vitro in the presence or absence of hES-MSCs and BM-MSCs. Under the Th1 condition, differentiation of naive $\mathrm{CD} 4^{+}$T cells into Th1 $\left(\mathrm{CD} 4^{+} / \mathrm{IFN} \gamma^{+}\right)$cells was reduced by hES-MSCs, but was unaffected or even enhanced by different BM-MSC lines (Figure 4C, upper panels). Interestingly, even the BM-MSC line (BMMSC\#6) that gave a modest but significant therapeutic 

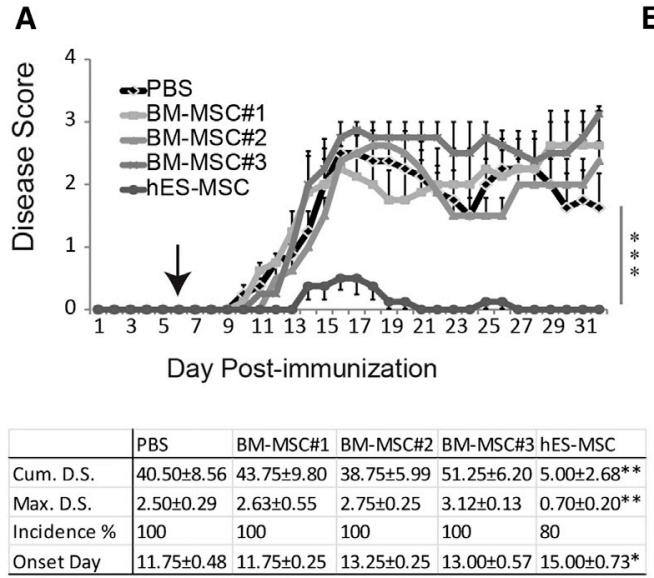

B
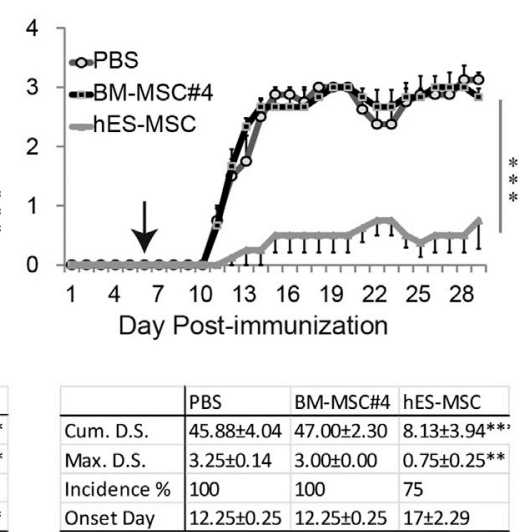

C

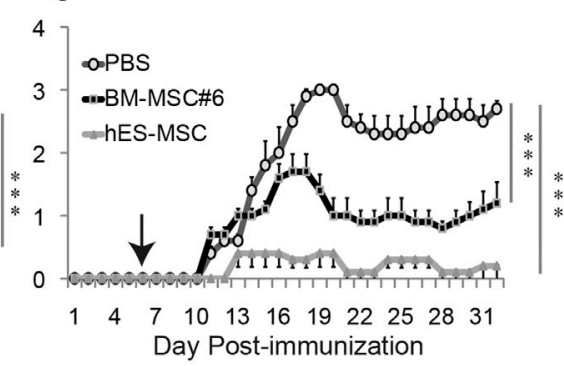

\begin{tabular}{|l|lll|}
\hline & PBS & BM-MSC\#6 & hES-MSC \\
\hline Cum. D.S. & $47.8 \pm 4.05$ & $23.5 \pm 4.63^{* *}$ & $5.2 \pm 2.58^{* * *}$ \\
\hline Max. D.S. & $3.0 \pm 0$ & $1.7 \pm 0.30^{*}$ & $0.4 \pm 0.19^{* * *}$ \\
\hline Incidence \% & 100 & 100 & 60 \\
\hline Onset Day & $11.2 \pm 0.2$ & $11.2 \pm 0.2$ & $13 \pm 0 * * *$ \\
\hline
\end{tabular}

D
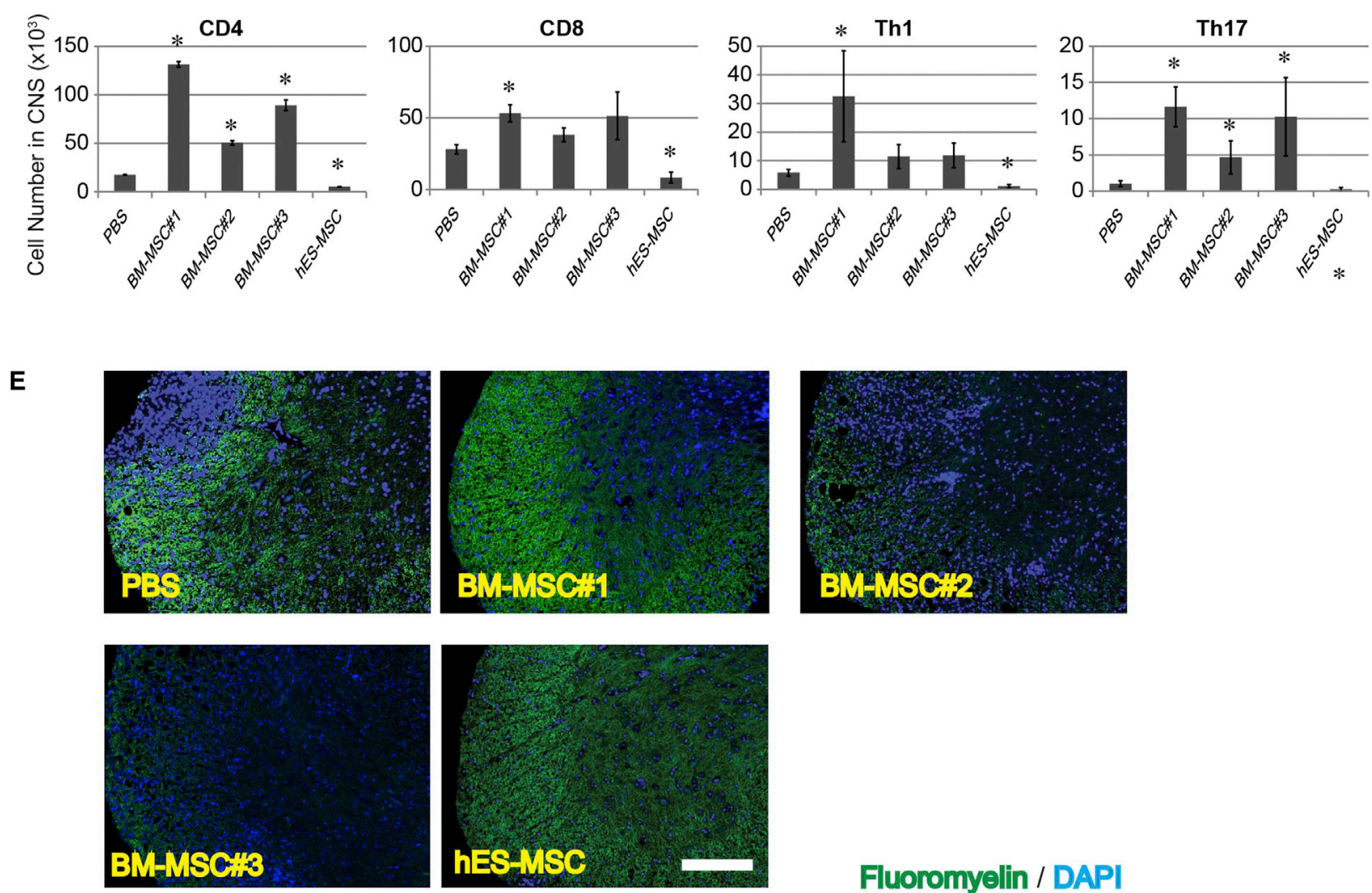

\section{Fluoromyelin / DAPI}

Figure 3. hES-MSCs Have a Stronger EAE-Inhibitory Effect In Vivo than BM-MSCs

(A-C) Disease scores of MOG $_{35-55}$ /CFA-immunized mice treated with PBS, $10^{6} \mathrm{BM}-\mathrm{MSC}$, or $10^{6} \mathrm{hES}$-MSCs (MA09) at day $6 . \mathrm{n}=4-5$ per group, $* * * p<0.001$ by Mann-Whitney for comparison with the PBS control. Error bar, SEM. The table below shows a comparison of cumulative disease score (Cum. D.S.), maximum disease score (Max. D.S.), disease incidence, and disease onset day.

(D) Total numbers of $\mathrm{CD}^{+}, \mathrm{CD}^{+}$, Th1, and Th17 cells in the CNS of $\mathrm{MOG}_{35-55}$ /CFA-immunized mice treated with PBS, BM-MSCs, or hES-MSCs on day 32 postimmunization. Lymphocytes purified from the CNS were analyzed via flow cytometry for numbers of $\mathrm{CD}_{4}^{+}$and $\mathrm{CD} 8^{+}$cells (left two panels) or $\mathrm{IL}^{-17^{+}}$and IFN $\gamma^{+}$cells (intracellular stained) poststimulation with 12-0-tetradecanoylphorbol-13-acetate (TPA) and ionomycin (right two panels). $n=4,{ }^{*} p<0.05,{ }^{* *} p<0.01$. Error bar, SD; $p$ values were determined using two-tailed unpaired Student's t test. 
response in the prophylactic EAE model could not effectively reduce Th1 differentiation in this in vitro assay (Figure 4C, upper last panel). Under the Th17 differentiation condition, both hES-MSCs and BM-MSCs reduced the differentiation of Th17 $\left(\mathrm{CD} 4^{+} / \mathrm{IL} 17^{+}\right)$cells (Figure $4 \mathrm{C}$, lower panels). However, under the same Th17-inducing conditions, BM-MSCs, but not hES-MSCs, significantly increased the percentage of $\mathrm{IFN}^{+} / \mathrm{IL}^{-} 7^{-}$(i.e., Th1) cells (Figure 4C, lower panels). Collectively, these results show that hES-MSCs effectively dampen differentiation of both Th1 and Th17 in vitro, and, surprisingly, BM-MSCs promote Th1 differentiation under a Th17-inducing environment.

BM-MSCs Express Higher Levels of IL-6 than hES-MSC, and IL-6 Blockage Enhances the Disease-Modifying

Effects of BM-MSCs in the EAE Model

Many factors have been reported to mediate the immunomodulatory and/or neuroprotective effects of MSCs (Uccelli and Prockop, 2010). We conducted a microarray analysis to identify differences in the expression of these factors between BM-MSCs and hES-MSCs. The overall expression profiles of the hES- and BM-MSC samples were similar (data not shown); however, a small set of genes was expressed differentially. Among these, IL6 appeared to be much more highly expressed in BM-MSCs than in hES-MSCs. Multiple methods, including quantitative RTPCR (qRT-PCR; Figure 5A), intracellular flow cytometry (Figure 5B), and cytokine antibody arrays (Figure 5C), confirmed this finding.

Upon IFN $\gamma$ stimulation, the percentage of IL-6-expressing hES-MSCs did not change; however, the percentage of IL-6-expressing BM-MSC nearly doubled (Figure 5D). We also tested MSC production of IL-6 following coculture with stimulated PBMC/T cells, as the latter produce high levels of IFN $\gamma$ and TNF- $\alpha$ upon stimulation. IL- 6 mRNA expression levels increased for both BM-MSCs and hESMSCs after coculture, yet the levels in BM-MSC were still $\geq 10$ times higher than in hES-MSCs (Figure S4A). This large difference in IL-6 secretion was a rather unique observation because the expression levels of other secreted cytokines did not differ dramatically between the two cell types (data not shown).

Since IL-6 has been found to enhance T cell differentiation (Dienz and Rincon, 2009), we sought to determine the effects of MSC-secreted human IL-6 on mouse T cell differentiation. First, we confirmed that human IL- 6 works just as well as mouse IL- 6 at an equivalent dose for directing mouse Th17 differentiation (Figure S4B). Next, under Th0 conditions (i.e., without any mouse cytokines), we found that an anti-human-IL-6 neutralizing antibody with no mouse cross-reactivity (clone MQ2-13A5) reduced the Th1-promoting effects of BM-MSCs on mouse $\mathrm{T}$ cells by $\sim 23 \%-50 \%$ (Figure $5 \mathrm{E}$ ). Lastly, we observed that $\mathrm{MOG}_{35-55} / \mathrm{CFA}$-immunized mice treated with human BMMSCs plus the same anti-hIL-6 antibody showed a significant reduction in EAE disease severity relative to the control group (Figure 5F). This effect was specific for IL-6 produced by the BM-MSCs, as IL- 6 antibody alone (no MSCs) or BM-MSCs plus isotype control antibody failed to reduce the EAE disease score significantly. These data suggest that high IL-6 expression by human BM-MSCs contributes to the inability of these cells to modulate EAE disease severity.

Both hES-MSCs and BM-MSCs Home to the Spinal Cord, but Only hES-MSCs Successfully Extravasate into Inflamed Tissue

To determine whether hES- and BM-MSCs home to the injured CNS, we used the constitutively GFP-expressing hESC line "Envy" and GFP-labeled human BM-MSCs (Hofstetter et al., 2002) in $\mathrm{MOG}_{35-55} / \mathrm{CFA}$-immunized mice. Cells were injected on day 6 after immunization and spinal cords were analyzed 8 days later (day 14 postimmunization), a time point when disease scores for the $\mathrm{GFP}^{+} \mathrm{BM}$ MSC-injected mice and PBS controls were $~ 1.5-2.0$, and scores for the $\mathrm{GFP}^{+} \mathrm{hES}$-MSC were effectively 0 (Figure $6 \mathrm{~A}$ ). Both BM-MSCs and hES-MSCs homed to the spinal cord in mice subjected to EAE (Figure 6B), yet the vascular association patterns of the two types of MSC were vastly different. At day $14, \mathrm{GFP}^{+}$hES-MSCs were immunolocalized in the parenchyma adjacent to spinal cord venules, indicating the ability of these cells to penetrate and move beyond the vasculature to enter the parenchyma (Figure $6 \mathrm{~B}$, top row). In marked contrast, $\mathrm{GFP}^{+} \mathrm{BM}$-MSCs appeared to remain closely associated with the parenchymal vessels, seemingly trapped inside the microvascular lumen and/or confined to the perivascular space, and incapable of breaching the bipartite complex of endothelial and parenchymal basement membranes (Owens et al., 2008; Paul et al., 2014) to enter the CNS tissue (Figure 6B, second row). This apparent retarded migration of BM-MSCs persisted even at day 18, when the disease is more severe and both tight junctions and the basement membrane complex of the BBB have been shown to be grossly disrupted (Figure 6B, third row) (Paul et al., 2013, 2014). No GFP

(E) Qualitative analysis of myelin content in spinal cord cross-sections of MOG $_{35-55} /$ CFA-immunized mice treated with PBS, BM-MSCs, or hES-MSCs using FluoroMyelin staining (green) and counterstained with DAPI (blue) for infiltration of nucleated cells. Scale bar, $350 \mu \mathrm{m}$. The regions shown are from the lumbar level of the spinal cord.

See also Figure S3. 
A

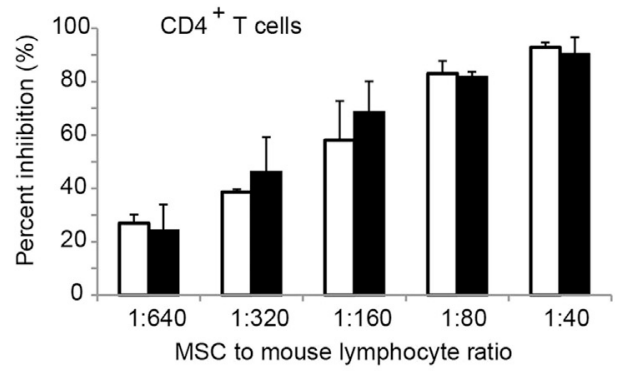

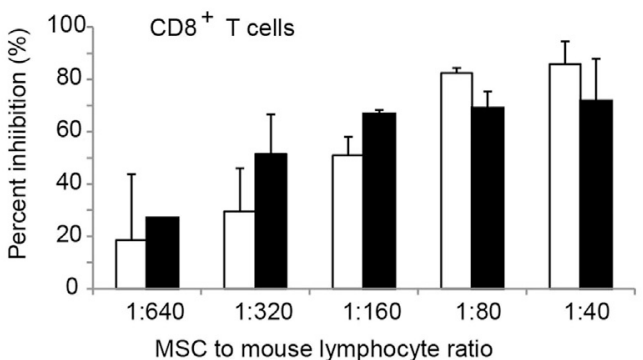

hES-MSC

B

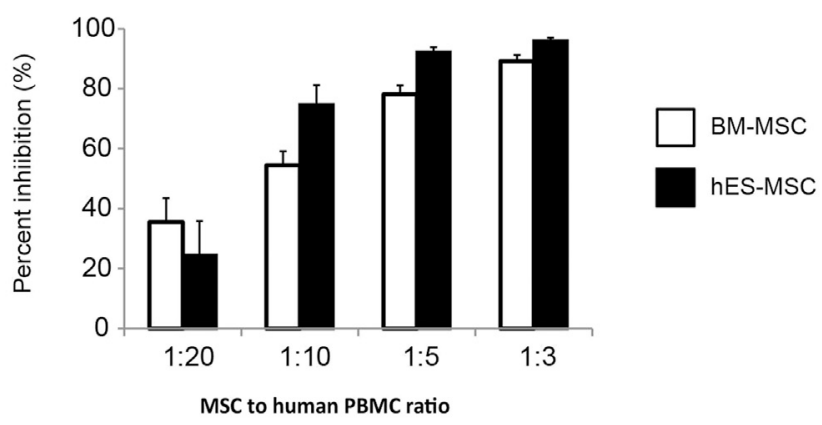

C Control hES-MSC

BM-MSC\#2
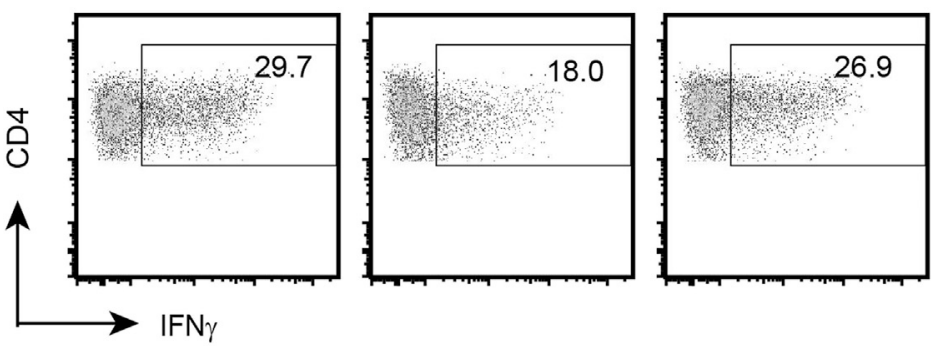

BM-MSC\#3

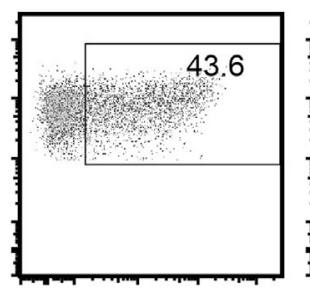

0.2
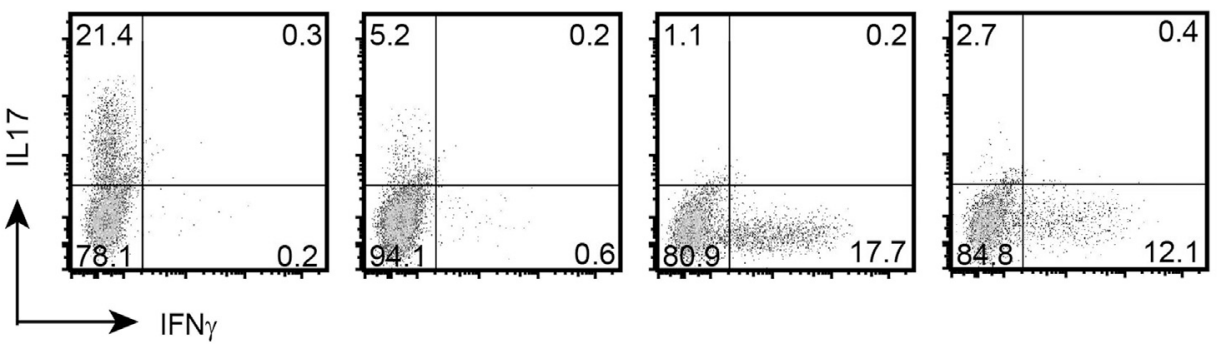

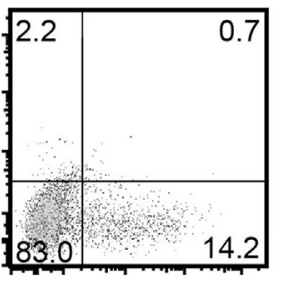

Th17

Condition

Figure 4. Effects of hES-MSCs and BM-MSCs on T Cell Functions In Vitro

(A) hES-MSCS (MA09) or hBM-MSC\#7 were cocultured with $1 \times 10^{5}$ CFSE-labeled mouse lymphocytes stimulated with anti-CD3/CD28 at various MSC/lymphocyte ratios. After 3 days, the proportion of proliferating $\mathrm{CD}^{+}$(left panel) or $\mathrm{CD} 8^{+}$(right panel) T cells was measured by CFSE dilution using flow cytometry. The percent inhibition of T cell proliferation is relative to T lymphocytes stimulated in the absence of MSCs. Lymphocytes from three individual mice were tested and results are average \pm SD.

(B) Mitotically inactivated hES-MSCS (MA09) or BM-MSCS were incubated with CFSE-labeled human PBMC at the indicated ratios and stimulated with $2.5 \mathrm{ng} / \mathrm{ml}$ PHA. Bars represent the average of three different BM-MSC lines and two independent hES-MSC (MA09) clones \pm SD.

(C) hES-MSCs (MA09) or BM-MSCs were incubated with mouse naive CD4 ${ }^{+} \mathrm{T}$ cells at a ratio of 1:10, followed by Th1 or Th17 differentiation for 5 days. IFN $\gamma^{+}$and $\mathrm{IL}-17^{+} \mathrm{CD}^{+} \mathrm{T}$ cells were detected via intracellular flow cytometry staining after TPA/ionomycin stimulation. Data represent four independent experiments. 
signals could be detected in control mice receiving PBS injection alone (Figure 6B, bottom row). Movies S1 and S2, which were made from confocal z-stack reconstructions of the data sets in Figure $6 \mathrm{~B}$, provide a magnified 3D perspective of hES-MSC and BM-MSC distributions, respectively. Figure $6 \mathrm{C}$ shows a schematic detailing the regions of spinal cord selected for analysis in Figure 6B.

\section{DISCUSSION}

In this study, we have shown that multiple hES-MSC lines significantly attenuated disease scores in a mouse EAE model of MS. In stark contrast, only one out of eight independently derived human BM-MSC lines displayed a marginal effect in the prophylactic-treatment EAE model. Our analysis of IL- 6 expression and migration of the MSCs suggests that the superior disease-altering effects of hES-MSCs may be related in part to the lower expression of IL-6 and the greater ability of hES-MSCs to extravasate the $\mathrm{BBB} / \mathrm{BSCB}$ and migrate into inflamed CNS tissue relative to BM-MSCs.

In examining the effects of hES-MSCs on EAE induction, we observed that preonset treatment of $\mathrm{MOG}_{35-55} / \mathrm{CFA}$ immunized mice was more effective in attenuating disease scores than postonset treatment. This is not surprising, since preonset treatment begins before the development of severe demyelination, axonal damage, or inflammatory cell infiltration. Costaining for MBP and NF in day 32 lumbar spine sections of treated animals showed that hES-MSC treatment protected against demyelination without affecting the number of surviving axons. These results do not rule out the possibility that hES-MSCs may also contribute to remyelination of axons that have already lost their myelin. This remains to be elucidated by further investigations examining whether and how hES-MSCs may contribute to neural regeneration. Future studies in the postonset model will also be needed to address whether larger doses and/or repeated injections of cells could enhance the therapeutic effects. Of note, irradiated hESMSCs were also effective in reducing the EAE disease score and had the same lifespan in vivo as their nonirradiated counterparts. Thus, irradiation of cells may provide an important clinical benefit by reducing concerns about the tumorigenic potential of MSCs.

The muted in vivo efficacy of BM-MSCs that we observed is consistent with previous reports that showed only mild (Gordon et al., 2008; Zhang et al., 2005) or negligible (Payne et al., 2013) effects in the EAE mouse model. Interestingly, BM-MSC\#6, the BM-MSC line that caused a modest reduction in EAE disease scores in the preonset model at passage 2 (Figure 3C), failed to show any therapeutic effects when used at passage 4 during the anti-iL- 6 antibody experiment (Figure 5F). On the other hand, hES-MSCs consistently resulted in large reductions in the disease score of $\mathrm{MOG}_{35-55} / \mathrm{CFA}$-immunized mice when used from passage 2 to 5 . This indicates that the therapeutic capacity of BM-MSCs may be more vulnerable to extended in vitro culture than that of hES-MSCs.

It has been reported that BM-MSCs derived from frozen MNCs have less immunosuppressive effects than those derived from fresh MNCs (Samuelsson et al., 2009). We derived different BM-MSC lines from both frozen MNCs and fresh (never frozen) whole BM. Both types had similarly negligible effects in the EAE model when administered prophylactically (Figures 3A, 3B, and S3F). One BMMSC line (\#8), derived from fresh BM, did show a modest efficacy in the EAE model when administered postonset (Figure S3I), but this effect was not as great as that of hESMSCs (Figure 1D). These data suggest that variability among human BM-MSC lines derived from different donors could influence the efficacy of these cells, whereas hES-MSC lines consistently exhibit a greater EAE-inhibitory effect than human BM-MSC lines.

In this study, we found that IL-6 was much more highly expressed in BM-MSCs than in hES-MSCs in both the basal and IFN $\gamma$-stimulated states. Elevated IL-6 levels have been found in blood and brain tissue from MS patients (Patanella et al., 2010), and site-specific production of IL-6 in the CNS can enhance inflammation in EAE (Quintana et al., 2009). Mice lacking IL- 6 receptor $\alpha$ are resistant to EAE (Leech et al., 2013), and an IL-6-neutralizing antibody can reduce symptoms in EAE mice (Gijbels et al., 1995). Thus, higher levels of human IL-6 secretion by BM-MSCs relative to hES-MSCs may contribute to functional differences in the treatment of EAE. This idea is supported by our data showing that blocking human IL-6 with a neutralizing antibody partially rescues the disease-modifying effects of BM-MSC in the EAE model (Figure 5F). It is important to note that the anti-hIL- 6 antibody in this study does not inhibit the endogenous mouse IL- 6 . This suggests that human IL-6 produced by the BM-MSCs may act in an autocrine or paracrine manner on the BM-MSCs themselves and/or on surrounding cells, with the net effect of limiting BM-MSC therapeutic activity in EAE.

Recent reports have noted that MSCs can actually promote the differentiation of proinflammatory $\mathrm{T}$ cells in certain permissive environments (Carrión et al., 2011; Darlington et al., 2010). Consistently, in vivo and in comparison with PBS controls, we observed reduced CNS infiltration of Th1 and Th17 cells with hES-MSC treatment, but increased CNS infiltration of Th1 and Th17 cells with BM-MSC treatment. In vitro and in contrast to hES-MSCs, we found that BM-MSCs skewed T cell differentiation to a Th1 phenotype under both Th0 (nonpolarizing) and Th17 conditions. It is possible that certain factors that are 
A

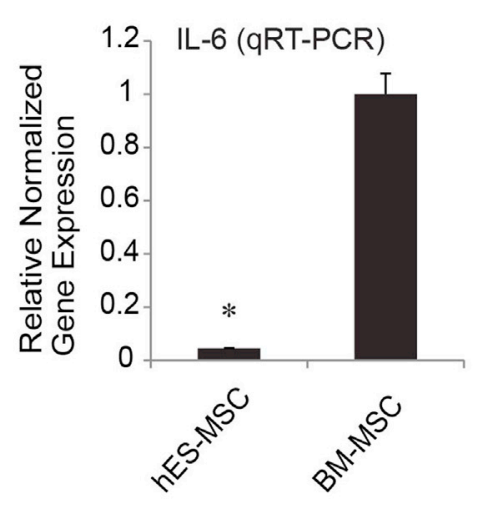

C

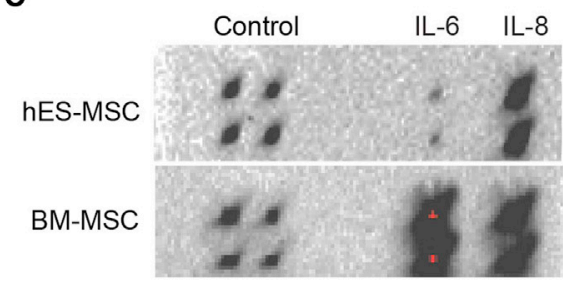

B

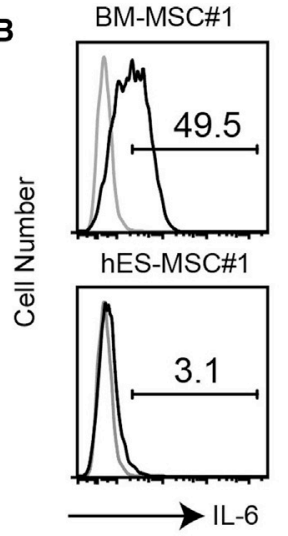

D

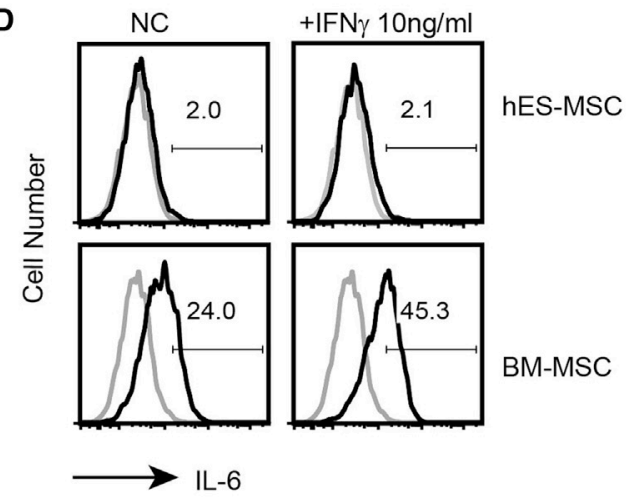

E

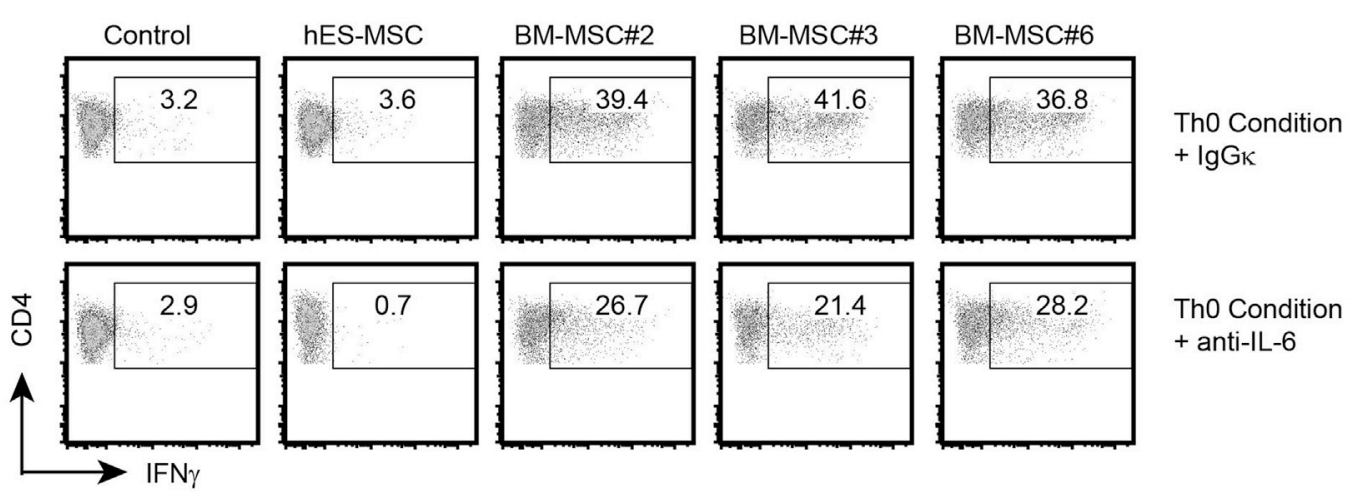

F

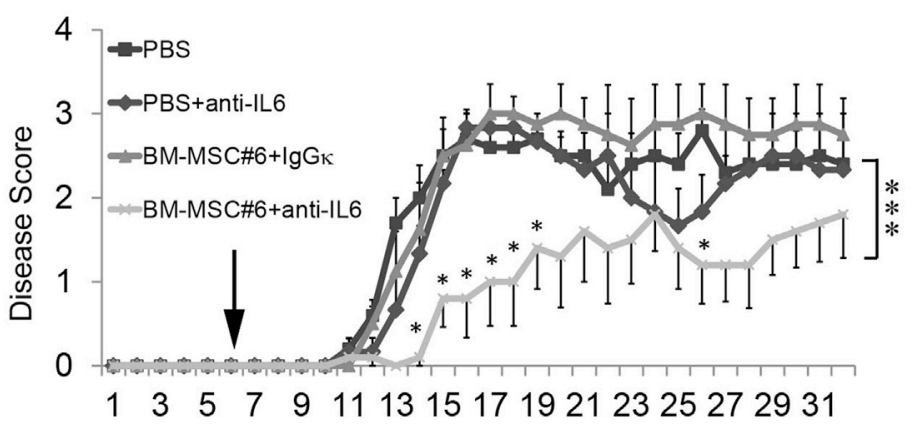

\begin{tabular}{|c|c|c|c|c|}
\hline & PBS & anti-IL-6 & BM-MSC+lgGK & BM-MSC+anti-IL-6 \\
\hline Cum. D.S. & $56.3 \pm 7.42$ & $49.33 \pm 8.29$ & $63.38 \pm 8.80$ & $30.4 \pm 10.06 * *$ \\
\hline Max. D.S. & $3.0 \pm 0.32$ & $3 \pm 0$ & $3.5 \pm 0.29$ & $2.2 \pm 0.37 *$ \\
\hline Incidence \% & 100 & 100 & 100 & 100 \\
\hline Onset Day & $11.8 \pm 0.37$ & $12.667 \pm 0.88$ & $12.25 \pm 0.25$ & $15.0 \pm 1.26$ * \\
\hline
\end{tabular}

(legend on next page) 
highly produced by human BM-MSCs, but not hES-MSCs, can trigger Th1 differentiation, thus overriding Th17 differentiation under defined in vitro conditions (Lazarevic et al., 2011). An anti-human IL-6 antibody was able to partially reverse the effect under Th0 conditions (Figure 5E), but not Th17 conditions (data not shown), presumably because the presence of abundant exogenous mouse IL-6, which was added for Th17 induction, could not be neutralized by the anti-human IL- 6 antibody. Together, these results suggest that high IL-6 secretion by BM-MSCs may impact the local cytokine milieu and augment the overall inflammatory response, resulting in a striking difference in Th1/Th17 CNS infiltration between hES-MSC- and BM-MSC-treated mice.

Lastly, we observed that both GFP-labeled hES-MSCs and BM-MSCs homed to the CNS microvasculature, but only hES-MSCs that showed a high therapeutic potential had the capacity to effectively extravasate and migrate into the parenchyma. This raises the possibility that therapeutic efficacy and MSC extravasation are somehow mechanistically linked. Such a therapeutic requirement for MSCs to extravasate during EAE is consistent with evidence that these cells can downregulate proinflammatory effector functions of parenchymal microglia (Figure 1B; Lee et al., 2012; Sheikh et al., 2011). The lack of penetration into the CNS tissue by BM-MSCs was further remarkable in that it persisted through late disease, a time when severe BBB disruption has been shown to occur (Paul et al., 2013 , 2014). Since such a compromised BBB might be expected to facilitate cellular entry during EAE (Lanz et al., 2013), this suggests that hES-MSCs might uniquely express specific transendothelial migratory signals or properties.
BM-MSCs, in turn, might lack one or more of the minimal requirements for effective migration across even a structurally attenuated CNS microvascular endothelium and/or surrounding basement membrane complex. It is significant that in vitro culture and expansion of MSCs have both been implicated as factors that impair homing and transendothelial migration (De Becker et al., 2007; Rombouts and Ploemacher, 2003). A priori, BM-MSCs could be more sensitive to these factors than hES-MSCs, which could at least partially explain the differences in therapeutic efficacy observed between the two MSC types. Determining the dynamic changes and differences in expression of adhesion molecules, chemokines/chemokine receptors, and matrix metalloproteinase between hES-MSCs and BM-MSCs in situ will be critical for delineating the molecular requirements for MSC extravasation and efficacy (De Becker et al., 2007; Teo et al., 2012). In situ gene-expression profiling of both MSC types is currently under investigation and should shed further light on the mechanism(s) responsible for the unique therapeutic efficacy of hES-MSCs in EAE.

\section{EXPERIMENTAL PROCEDURES}

\section{Culture of hESCs and Generation of hES-MSCs}

hESC lines were cultured either on Matrigel in TeSR1 medium or on mouse embryonic fibroblasts in Dulbecco's modified Eagle's medium/F12 + 20\% knockout serum replacement $+10 \mathrm{ng} / \mathrm{ml}$ basic fibroblast growth factor. hES-MSCs were generated as described previously (Kimbrel et al., 2014). Only hES-MSCs at $\leq 5$ passages were used throughout the study. The use of hESCs in this study was approved by the Stem Cell Research Oversight Committee of the University of Connecticut (\#2012-005).

Figure 5. BM-MSCs Express Higher Levels of IL-6 than hES-MSCs, and a Neutralizing IL-6 Antibody Reduces the Influence of BMMSCs on T Cells and Disease Scores

(A) qRT-PCR for IL-6 in BM-MSCs versus hES-MSCs. Bars represent the average of three independent experiments \pm SD. * $p<0.05$ (the $\mathrm{p}$ value was determined using two-tailed unpaired Student's $t$ test).

(B) Intracellular flow cytometry showing the percentage of IL-6-expressing cells (black line) and isotype control (gray line) among BMMSC from three different donors and hES-MSCs from three hESC lines (MA09, CT2, and H9).

(C) Cytokine antibody arrays showing the level of IL-6 and IL-8 proteins in conditioned medium from hES-MSC or BM-MSC cultures. Cytokine antibodies and controls are spotted in duplicate and labeled next to the corresponding spots. Images are representative of at least five independent experiments.

(D) Intracellular flow cytometry showing the percentage of IL-6-expressing cells (black line) and isotype control (gray line) among hESMSCs or BM-MSCs treated $\pm 10 \mathrm{ng} / \mathrm{ml}$ IFN $\gamma$ for $12 \mathrm{hr}$. NC, negative control.

(E) Intracellular flow cytometry measuring the percentage of $\mathrm{CD}^{+}$and IFN $\gamma^{+}$mouse $\mathrm{T}$ cells arising from naive T cells after coculture with hES-MSC (MA09) or BM-MSCs from three different donors under Th0 conditions. The MSC/T cell ratio was 1:10. IgGк isotype control (upper panels) or anti-human IL-6 neutralizing antibody (clone MQ2-13A5) was added to determine the effect of blocking IL-6 on BM-MSCinduced production of IFN $\gamma$ from $\mathrm{T}$ cells (i.e., Th1 differentiation) (lower panels).

(F) Mean disease scores of mice immunized with MOG $_{35-55}$ and treated with PBS or $1 \times 10^{6}$ human BM-MSC on day 6 . Anti-human IL-6 neutralizing antibody (clone MQ2-13A5, $12.5 \mathrm{mg} / \mathrm{kg} /$ day), IgGK isotype control antibody (12.5 mg/kg/day) or PBS was administered i.v. on days 6 and $7 .{ }^{* *} p<0.001$ by Mann-Whitney for BM-MSC\#6+anti-iL- 6 versus PBS control ( $n=5$ per group). Error bar, SEM. The table on the right shows a comparison of cumulative disease score (Cum. D.S.), maximum disease score (Max. D.S.), disease incidence, and disease onset day.

See also Figure $\mathbf{S 4}$. 
A

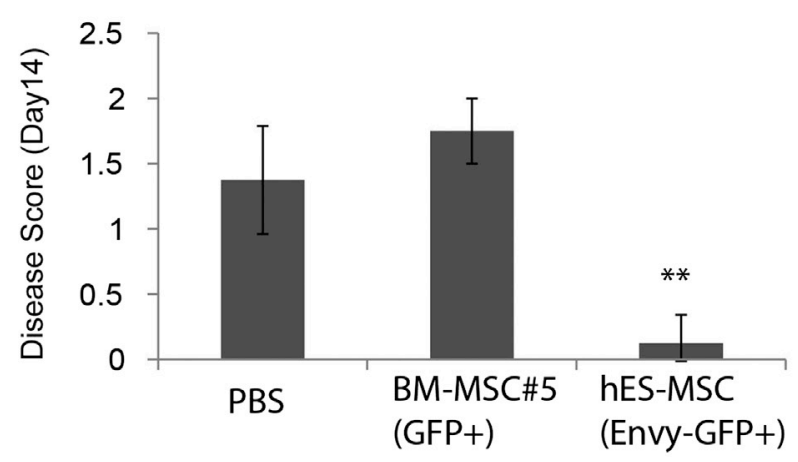

C

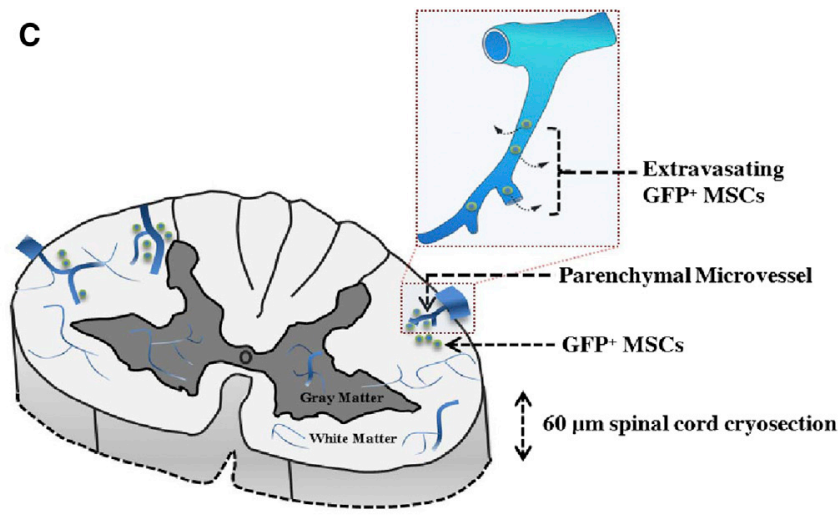

B
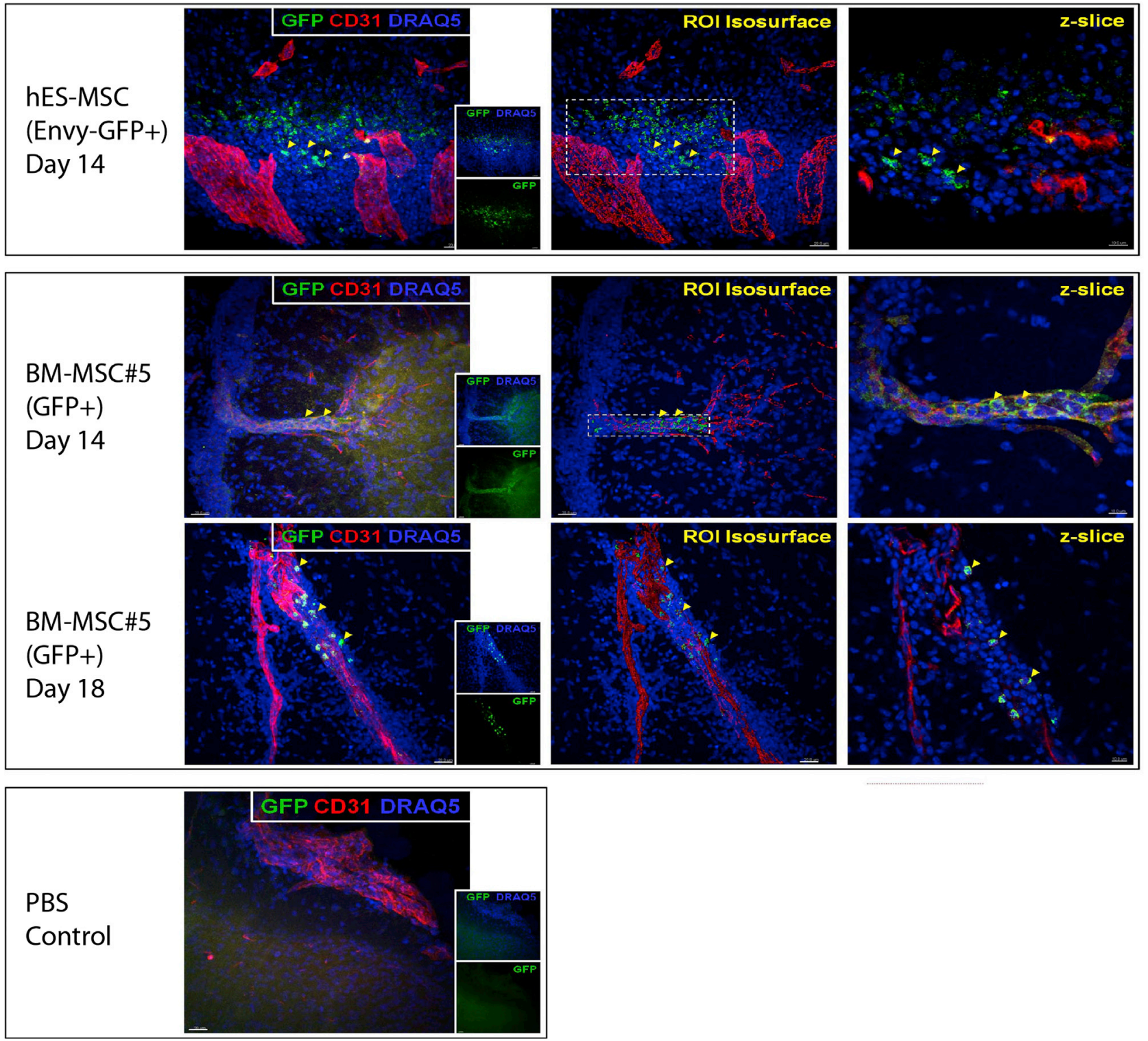


\section{Animal Model of MS}

The mouse EAE model was induced as previously described (Stromnes and Goverman, 2006). In brief, C57BL/6 mice were subcutaneously injected with an emulsion of $\mathrm{MOG}_{35-55}$ peptide, $\mathrm{CFA}$, and pertussis toxin contained in the EAE induction kit from Hooke Laboratories (Cat. No. EK-0114). BM-MSCs or hES-MSCs at $1 \times 10^{6}$ cells/mouse or PBS (a vehicle control) were i.p. injected on day 6 (for preonset) or 18 (for postonset) after the immunization. Disease score was monitored every day for up to 31 or more days as follows: 0 , no sign of disease; 1 , loss of tone in the tail; 2 , partial hind limb paralysis; 3, complete hind limb paralysis; 4, front limb paralysis; and 5, moribund (Stromnes and Goverman, 2006). For some experiments, cumulative and maximal disease scores were also calculated, and disease incidence and disease onset day were recorded. Injection and scoring were performed double-blinded in Figure S3F. All animal studies were approved by and performed in accordance with policies of the Institutional Animal Care and Use Committee of the University of Connecticut Health Center.

\section{Tracking of $\mathrm{GFP}^{+}$MSCs in Perivascular Regions in} the CNS

Spinal cord tissue was prepared as described previously (Paul et al., 2013). In brief, after perfusion/fixation, spinal cords were harvested by laminectomy and cryosectioned for immunostaining. Anti-GFP-Alexa 488, anti-CD31 (BD Bioscience), and Alexa 555 secondary antibody (Life Technologies) were used to detect $\mathrm{GFP}^{+}$ and endothelial cells, and DRAQ5 (Biostatus) was used to visualize the nuclei. Sections were then mounted in Mowiol and confocal z-stacks were acquired at $1 \mu \mathrm{m}$ increments between $\mathrm{z}$ slices, following a multitrack scan, using a Zeiss LSM 510 Meta confocal microscope. Images were analyzed with Imaris suite version 7.1 software (Bitplane). The GFP channel was isosurface rendered to provide better spatial perspective for visualizing $\mathrm{GFP}^{+}$cells.

\section{Statistical Analysis}

The EAE clinical disease scores for each group were graphed as the mean \pm SEM for each day of the study. Differences in EAE disease scores between groups were analyzed using the nonparametric Mann-Whitney unpaired U test, and individual time-point differences were analyzed using two-way ANOVA with Bonferroni's posttest. Percentage data for CNS-infiltrated T cells were arcsine transformed prior to analysis. EXCEL and Prism 6.0 (GraphPad) software was used for statistical analysis, and $\mathrm{p}<0.05$ was consid- ered to be statistically significant $\left({ }^{*} \mathrm{p}<0.05,{ }^{* *} \mathrm{p}<0.01,{ }^{* *} \mathrm{p}<\right.$ 0.001).

\section{SUPPLEMENTAL INFORMATION}

Supplemental Information includes Supplemental Experimental Procedures, four figures, and two movies and can be found with this article online at http://dx.doi.org/10.1016/j.stemcr. 2014.04.020.

\section{AUTHOR CONTRIBUTIONS}

X.W., E.A.K., S.-J.L., J.S.P., S.C., and R.-H.X. conceived and designed the research. X.W., E.A.K., K.I., D.P., A.S.L., J.C., N.A.K., and G.Y. performed the experiments. X.W., E.A.K., D.P., A.S.L., J.S.P., S.C., R.L., and R.-H.X. analyzed the data and wrote the manuscript.

\section{ACKNOWLEDGMENTS}

We thank Dr. Lingzhao Cheng for the pLV-EF1 $\alpha$-Luc-IRES-Puro vector. Some of the MSC lines were provided by the Texas A\&M Health Science Center College of Medicine Institute for Regenerative Medicine at Scott \& White through a grant from the NCRR, NIH (P40RR017447). This work was supported by Connecticut Stem Cell Research grants 12-SCD-UCHC-01 and 13-SCD-UCHC01 to R.X.; 13-SCDIS-ISB-01 to R.X., J.S.P., and X.W.; and 10SCA21 to X.W. This work was also supported in part by grants from ImStem Biotechnology and Advanced Cell Technology. X.W. is a founder and employee of, A.S.L. is an employee of, and R.-H.X. is a founder and scientific advisor of ImStem Biotechnology. E.A.K., J.C., N.A.K., G.Y., S.-J.L., and R.L. are employees of Advanced Cell Technology.

Received: April 14, 2014

Revised: April 24, 2014

Accepted: April 29, 2014

Published: June 5, 2014

\section{REFERENCES}

Auletta, J.J., Bartholomew, A.M., Maziarz, R.T., Deans, R.J., Miller, R.H., Lazarus, H.M., and Cohen, J.A. (2012). The potential of mesenchymal stromal cells as a novel cellular therapy for multiple sclerosis. Immunotherapy 4, 529-547.

Figure 6. GFP $^{+}$hES-MSCs, but not GFP ${ }^{+}$BM-MSCs, Effectively Extravasate from Inflamed CNS Venules and Migrate into CNS Parenchyma of $\mathrm{MOG}_{35-55}$ /CFA-Immunized Mice

$\mathrm{MOG}_{35-55}$ /CFA-immunized mice were treated with GFP+ hESC-MSC (Envy), GFP+ BM-MSC, or PBS control on day 6.

(A) Average disease scores on the day of spinal cord harvest (day 14). $n=4$ mice per group. Error bar, SD; ${ }^{*} p<0.01$ was determined using two-tailed unpaired Student's t test.

(B) 3D confocal data sets from spinal cord cryosections $(60 \mu \mathrm{m})$ of MSC-injected MOG $_{35-55} /$ CFA-immunized mice stained with anti-GFP (green) for MSC, anti-CD31 (red) for microvessels, and DRAQ5 (blue) for nuclei. Yellow arrowheads point to clusters of GFP ${ }^{+}$MSCs. Volumerendered $z$ stacks (left column), isosurface-rendered GFP+ ${ }^{+}$cells (middle column), and zoomed-in representative single $z$ slices to highlight the spatial location of GFP ${ }^{+}$cells relative to microvessels (right column) are shown respectively in each row. GFP ${ }^{+}$hES-MSCs at day 14 (top row), GFP BM-MSCs at day 14 (second row), GFP+ BM-MSCs at day 18 (third row), and PBS only (bottom row). Scale bar, $20 \mu \mathrm{m}$.

(C) Cartoon depicts microvessels from the dorsolateral white matter that were selected for analysis.

See also Movies S1 and S2. 
Bai, L., Lennon, D.P., Eaton, V., Maier, K., Caplan, A.I., Miller, S.D., and Miller, R.H. (2009). Human bone marrow-derived mesenchymal stem cells induce Th2-polarized immune response and promote endogenous repair in animal models of multiple sclerosis. Glia 57, 1192-1203.

Barberi, T., Willis, L.M., Socci, N.D., and Studer, L. (2005). Derivation of multipotent mesenchymal precursors from human embryonic stem cells. PLoS Med. 2, e161.

Barlow, S., Brooke, G., Chatterjee, K., Price, G., Pelekanos, R., Rossetti, T., Doody, M., Venter, D., Pain, S., Gilshenan, K., and Atkinson, K. (2008). Comparison of human placenta- and bone marrow-derived multipotent mesenchymal stem cells. Stem Cells Dev. 17, 1095-1107.

Brown, S.E., Tong, W., and Krebsbach, P.H. (2009). The derivation of mesenchymal stem cells from human embryonic stem cells. Cells Tissues Organs (Print) 189, 256-260.

Carrión, F., Nova, E., Luz, P., Apablaza, F., and Figueroa, F. (2011). Opposing effect of mesenchymal stem cells on Th1 and Th17 cell polarization according to the state of $\mathrm{CD} 4+\mathrm{T}$ cell activation. Immunol. Lett. 135, 10-16.

Chao, Y.X., He, B.P., and Tay, S.S. (2009). Mesenchymal stem cell transplantation attenuates blood brain barrier damage and neuroinflammation and protects dopaminergic neurons against MPTP toxicity in the substantia nigra in a model of Parkinson's disease. J. Neuroimmunol. 216, 39-50.

Connick, P., Kolappan, M., Crawley, C., Webber, D.J., Patani, R., Michell, A.W., Du, M.Q., Luan, S.L., Altmann, D.R., Thompson, A.J., et al. (2012). Autologous mesenchymal stem cells for the treatment of secondary progressive multiple sclerosis: an open-label phase 2a proof-of-concept study. Lancet Neurol. 11, 150-156.

Costa, M., Dottori, M., Ng, E., Hawes, S.M., Sourris, K., Jamshidi, P., Pera, M.F., Elefanty, A.G., and Stanley, E.G. (2005). The hESC line Envy expresses high levels of GFP in all differentiated progeny. Nat. Methods 2, 259-260.

Darlington, P.J., Boivin, M.N., Renoux, C., François, M., Galipeau, J., Freedman, M.S., Atkins, H.L., Cohen, J.A., Solchaga, L., and BarOr, A. (2010). Reciprocal Th1 and Th17 regulation by mesenchymal stem cells: Implication for multiple sclerosis. Ann. Neurol. $68,540-545$.

De Becker, A., Van Hummelen, P., Bakkus, M., Vande Broek, I., De Wever, J., De Waele, M., and Van Riet, I. (2007). Migration of culture-expanded human mesenchymal stem cells through bone marrow endothelium is regulated by matrix metalloproteinase- 2 and tissue inhibitor of metalloproteinase-3. Haematologica 92, 440-449.

Dienz, O., and Rincon, M. (2009). The effects of IL-6 on CD4 T cell responses. Clin. Immunol. 130, 27-33.

Djouad, F., Plence, P., Bony, C., Tropel, P., Apparailly, F., Sany, J., Noël, D., and Jorgensen, C. (2003). Immunosuppressive effect of mesenchymal stem cells favors tumor growth in allogeneic animals. Blood 102, 3837-3844.

Gijbels, K., Brocke, S., Abrams, J.S., and Steinman, L. (1995). Administration of neutralizing antibodies to interleukin-6 (IL-6) reduces experimental autoimmune encephalomyelitis and is asso- ciated with elevated levels of IL-6 bioactivity in central nervous system and circulation. Mol. Med. 1, 795-805.

Giuliani, M., Fleury, M., Vernochet, A., Ketroussi, F., Clay, D., Azzarone, B., Lataillade, J.J., and Durrbach, A. (2011). Long-lasting inhibitory effects of fetal liver mesenchymal stem cells on T-lymphocyte proliferation. PLoS ONE 6, e19988.

Gordon, D., Pavlovska, G., Glover, C.P., Uney, J.B., Wraith, D., and Scolding, N.J. (2008). Human mesenchymal stem cells abrogate experimental allergic encephalomyelitis after intraperitoneal injection, and with sparse CNS infiltration. Neurosci. Lett. 448, 71-73.

Gordon, D., Pavlovska, G., Uney, J.B., Wraith, D.C., and Scolding, N.J. (2010). Human mesenchymal stem cells infiltrate the spinal cord, reduce demyelination, and localize to white matter lesions in experimental autoimmune encephalomyelitis. J. Neuropathol. Exp. Neurol. 69, 1087-1095.

Gruenloh, W., Kambal, A., Sondergaard, C., McGee, J., Nacey, C., Kalomoiris, S., Pepper, K., Olson, S., Fierro, F., and Nolta, J.A. (2011). Characterization and in vivo testing of mesenchymal stem cells derived from human embryonic stem cells. Tissue Eng. Part A 17, 1517-1525.

Hofstetter, C.P., Schwarz, E.J., Hess, D., Widenfalk, J., El Manira, A., Prockop, D.J., and Olson, L. (2002). Marrow stromal cells form guiding strands in the injured spinal cord and promote recovery. Proc. Natl. Acad. Sci. USA 99, 2199-2204.

Hwang, N.S., Varghese, S., Lee, H.J., Zhang, Z., Ye, Z., Bae, J., Cheng, L., and Elisseeff, J. (2008). In vivo commitment and functional tissue regeneration using human embryonic stem cellderived mesenchymal cells. Proc. Natl. Acad. Sci. USA 105, 20641-20646.

Karussis, D., Karageorgiou, C., Vaknin-Dembinsky, A., GowdaKurkalli, B., Gomori, J.M., Kassis, I., Bulte, J.W., Petrou, P., Ben-Hur, T., Abramsky, O., and Slavin, S. (2010). Safety and immunological effects of mesenchymal stem cell transplantation in patients with multiple sclerosis and amyotrophic lateral sclerosis. Arch. Neurol. 67, 1187-1194.

Kimbrel, E.A., Kouris, N.A., Yavanian, G.J., Chu, J., Qin, Y., Chan, A., Singh, R.P., McCurdy, D., Gordon, L., Levinson, R.D., and Lanza, R. (2014). Mesenchymal stem cell population derived from human pluripotent stem cells displays potent immunomodulatory and therapeutic properties. Stem Cells Dev.

Klimanskaya, I., Chung, Y., Becker, S., Lu, S.J., and Lanza, R. (2006). Human embryonic stem cell lines derived from single blastomeres. Nature 444, 481-485.

Kyriakou, C., Rabin, N., Pizzey, A., Nathwani, A., and Yong, K. (2008). Factors that influence short-term homing of human bone marrow-derived mesenchymal stem cells in a xenogeneic animal model. Haematologica 93, 1457-1465.

Lalu, M.M., McIntyre, L., Pugliese, C., Fergusson, D., Winston, B.W., Marshall, J.C., Granton, J., and Stewart, D.J.; Canadian Critical Care Trials Group (2012). Safety of cell therapy with mesenchymal stromal cells (SafeCell): a systematic review and meta-analysis of clinical trials. PLoS ONE 7, e47559.

Lanz, T.V., Becker, S., Osswald, M., Bittner, S., Schuhmann, M.K., Opitz, C.A., Gaikwad, S., Wiestler, B., Litzenburger, U.M., Sahm, 
F., et al. (2013). Protein kinase $\mathrm{C} \beta$ as a therapeutic target stabilizing blood-brain barrier disruption in experimental autoimmune encephalomyelitis. Proc. Natl. Acad. Sci. USA 110, 14735-14740.

Lazarevic, V., Chen, X., Shim, J.H., Hwang, E.S., Jang, E., Bolm, A.N., Oukka, M., Kuchroo, V.K., and Glimcher, L.H. (2011). T-bet represses $\mathrm{T}(\mathrm{H}) 17$ differentiation by preventing Runx1-mediated activation of the gene encoding ROR $\gamma$ t. Nat. Immunol. 12, 96-104.

Lee, H.J., Lee, J.K., Lee, H., Carter, J.E., Chang, J.W., Oh, W., Yang, Y.S., Suh, J.G., Lee, B.H., Jin, H.K., and Bae, J.S. (2012). Human umbilical cord blood-derived mesenchymal stem cells improve neuropathology and cognitive impairment in an Alzheimer's disease mouse model through modulation of neuroinflammation. Neurobiol. Aging 33, 588-602.

Leech, M.D., Barr, T.A., Turner, D.G., Brown, S., O'Connor, R.A., Gray, D., Mellanby, R.J., and Anderton, S.M. (2013). Cutting edge: IL-6-dependent autoimmune disease: dendritic cells as a sufficient, but transient, source. J. Immunol. 190, 881-885.

Lu, S.J., Feng, Q., Caballero, S., Chen, Y., Moore, M.A., Grant, M.B., and Lanza, R. (2007). Generation of functional hemangioblasts from human embryonic stem cells. Nat. Methods 4, 501-509.

McFarland, H.F., and Martin, R. (2007). Multiple sclerosis: a complicated picture of autoimmunity. Nat. Immunol. 8, 913-919.

Mohyeddin Bonab, M., Yazdanbakhsh, S., Lotfi, J., Alimoghaddom, K., Talebian, F., Hooshmand, F., Ghavamzadeh, A., and Nikbin, B. (2007). Does mesenchymal stem cell therapy help multiple sclerosis patients? Report of a pilot study. Iran. J. Immunol. 4, 50-57.

Olivier, E.N., Rybicki, A.C., and Bouhassira, E.E. (2006). Differentiation of human embryonic stem cells into bipotent mesenchymal stem cells. Stem Cells 24, 1914-1922.

Owens, T., Bechmann, I., and Engelhardt, B. (2008). Perivascular spaces and the two steps to neuroinflammation. J. Neuropathol. Exp. Neurol. 67, 1113-1121.

Patanella, A.K., Zinno, M., Quaranta, D., Nociti, V., Frisullo, G., Gainotti, G., Tonali, P.A., Batocchi, A.P., and Marra, C. (2010). Correlations between peripheral blood mononuclear cell production of BDNF, TNF-alpha, IL-6, IL-10 and cognitive performances in multiple sclerosis patients. J. Neurosci. Res. 88, 1106-1112.

Paul, D., Cowan, A.E., Ge, S., and Pachter, J.S. (2013). Novel 3D analysis of Claudin-5 reveals significant endothelial heterogeneity among CNS microvessels. Microvasc. Res. 86, 1-10.

Paul, D., Ge, S., Lemire, Y., Jellison, E.R., Serwanski, D.R., Ruddle, N.H., and Pachter, J.S. (2014). Cell-selective knockout and 3D confocal image analysis reveals separate roles for astrocyte-and endothelial-derived CCL2 in neuroinflammation. J. Neuroinflammation 11, 10 .

Payne, N.L., Sun, G., McDonald, C., Layton, D., Moussa, L., Emerson-Webber, A., Veron, N., Siatskas, C., Herszfeld, D., Price, J., and Bernard, C.C. (2013). Distinct immunomodulatory and migratory mechanisms underpin the therapeutic potential of human mesenchymal stem cells in autoimmune demyelination. Cell Transplant. 22, 1409-1425.

Peron, J.P., Jazedje, T., Brandão, W.N., Perin, P.M., Maluf, M., Evangelista, L.P., Halpern, S., Nisenbaum, M.G., Czeresnia, C.E., Zatz, M., et al. (2012). Human endometrial-derived mesenchymal stem cells suppress inflammation in the central nervous system of EAE mice. Stem Cell Rev. 8, 940-952.

Pittenger, M.F., Mackay, A.M., Beck, S.C., Jaiswal, R.K., Douglas, R., Mosca, J.D., Moorman, M.A., Simonetti, D.W., Craig, S., and Marshak, D.R. (1999). Multilineage potential of adult human mesenchymal stem cells. Science 284, 143-147.

Pomper, M.G., Hammond, H., Yu, X., Ye, Z., Foss, C.A., Lin, D.D., Fox, J.J., and Cheng, L. (2009). Serial imaging of human embryonic stem-cell engraftment and teratoma formation in live mouse models. Cell Res. 19, 370-379.

Quintana, A., Müller, M., Frausto, R.F., Ramos, R., Getts, D.R., Sanz, E., Hofer, M.J., Krauthausen, M., King, N.J., Hidalgo, J., and Campbell, I.L. (2009). Site-specific production of IL-6 in the central nervous system retargets and enhances the inflammatory response in experimental autoimmune encephalomyelitis. J. Immunol. 183, 2079-2088.

Rombouts, W.J., and Ploemacher, R.E. (2003). Primary murine MSC show highly efficient homing to the bone marrow but lose homing ability following culture. Leukemia 17, 160-170.

Samuelsson, H., Ringdén, O., Lönnies, H., and Le Blanc, K. (2009). Optimizing in vitro conditions for immunomodulation and expansion of mesenchymal stromal cells. Cytotherapy 11, 129-136.

Sánchez, L., Gutierrez-Aranda, I., Ligero, G., Rubio, R., MuñozLópez, M., García-Pérez, J.L., Ramos, V., Real, P.J., Bueno, C., Rodríguez, R., et al. (2011). Enrichment of human ESC-derived multipotent mesenchymal stem cells with immunosuppressive and anti-inflammatory properties capable to protect against experimental inflammatory bowel disease. Stem Cells 29, 251-262.

Sheikh, A.M., Nagai, A., Wakabayashi, K., Narantuya, D., Kobayashi, S., Yamaguchi, S., and Kim, S.U. (2011). Mesenchymal stem cell transplantation modulates neuroinflammation in focal cerebral ischemia: contribution of fractalkine and IL-5. Neurobiol. Dis. 41, 717-724.

Stromnes, I.M., and Goverman, J.M. (2006). Active induction of experimental allergic encephalomyelitis. Nat. Protoc. 1, 18101819.

Teo, G.S., Ankrum, J.A., Martinelli, R., Boetto, S.E., Simms, K., Sciuto, T.E., Dvorak, A.M., Karp, J.M., and Carman, C.V. (2012). Mesenchymal stem cells transmigrate between and directly through tumor necrosis factor- $\alpha$-activated endothelial cells via both leukocyte-like and novel mechanisms. Stem Cells 30, 2472 2486.

Thomson, J.A., Itskovitz-Eldor, J., Shapiro, S.S., Waknitz, M.A., Swiergiel, J.J., Marshall, V.S., and Jones, J.M. (1998). Embryonic stem cell lines derived from human blastocysts. Science 282, 1145-1147.

Uccelli, A., and Prockop, D.J. (2010). Why should mesenchymal stem cells (MSCs) cure autoimmune diseases? Curr. Opin. Immunol. 22, 768-774.

Vodyanik, M.A., Yu, J., Zhang, X., Tian, S., Stewart, R., Thomson, J.A., and Slukvin, I.I. (2010). A mesoderm-derived precursor for mesenchymal stem and endothelial cells. Cell Stem Cell 7, 718-729. 
Wang, X., Lin, G., Martins-Taylor, K., Zeng, H., and Xu, R.H. (2009). Inhibition of caspase-mediated anoikis is critical for basic fibroblast growth factor-sustained culture of human pluripotent stem cells. J. Biol. Chem. 284, 34054-34064.

Weber, M.S., Menge, T., Lehmann-Horn, K., Kronsbein, H.C., Zettl, U., Sellner, J., Hemmer, B., and Stüve, O. (2012). Current treatment strategies for multiple sclerosis - efficacy versus neurological adverse effects. Curr. Pharm. Des. 18, 209-219.

Wong, R.S. (2011). Mesenchymal stem cells: angels or demons? J. Biomed. Biotechnol. 2011, 459510.

Yamout, B., Hourani, R., Salti, H., Barada, W., El-Hajj, T., AlKutoubi, A., Herlopian, A., Baz, E.K., Mahfouz, R., Khalil-Hamdan,
R., et al. (2010). Bone marrow mesenchymal stem cell transplantation in patients with multiple sclerosis: a pilot study. J. Neuroimmunol. 227, 185-189.

Zappia, E., Casazza, S., Pedemonte, E., Benvenuto, F., Bonanni, I., Gerdoni, E., Giunti, D., Ceravolo, A., Cazzanti, F., Frassoni, F., et al. (2005). Mesenchymal stem cells ameliorate experimental autoimmune encephalomyelitis inducing T-cell anergy. Blood $106,1755-1761$.

Zhang, J., Li, Y., Chen, J., Cui, Y., Lu, M., Elias, S.B., Mitchell, J.B., Hammill, L., Vanguri, P., and Chopp, M. (2005). Human bone marrow stromal cell treatment improves neurological functional recovery in EAE mice. Exp. Neurol. 195, 16-26. 